\title{
COMPARATIVE STUDY OF FRACTURE RESISTANCE OF DIFFERENT CERAMIC RESTORATIONS
}

\author{
Marwa Mohamed Mogahed, ${ }^{*}$ Mahmoud Abdelsalam Shakal ${ }^{* *}$ \\ and Fatma Ahmad Hasaneen***
}

\begin{abstract}
Objective: Comparison of fracture resistance of different ceramic restorations.

Methodology: 64 ceramic crowns were fabricated. They were divided into 4 equal groups according to the type of ceramic used (16 each). Group I: lithium disilicate, Group II: Zirconia reinforced lithium silicate, Group III: monolithic zirconia and Group IV: bilayered zirconia. Models of prepared teeth \#14 were used as abutments. Eight specimens from each group were subjected to thermocycling between $55^{\circ} \mathrm{C}$ and $5^{\circ} \mathrm{C}$. Fracture resistance was tested for the specimens. The load to fracture was recorded. Mean value for each group was calculated and differences between groups were tested for statistical significance. One fractured specimen from each group was scanned by scanning electron microscope to determine the failure mode.
\end{abstract}

Results: it was found that the highest fracture resistance mean value was recorded with monolithic zirconia group followed by lithium disilicate group then bilayered zirconia group while the lowest fracture resistance mean value was recorded for zirconia reinforced lithium silicate group and this was statistically significant. The four tested groups showed lower fracture resistance mean values after thermocycling. The fractography showed that surface defects were the origin of fracture in glass ceramic groups. While monolithic zirconia showed internal surface cracks.

Conclusions: Within the limitations of this study, the following conclusions can be drawn: monolithic zirconia has the highest fracture resistance among the tested groups, while zirconia reinforced lithium silicate group has the lowest fracture resistance. Thermal aging significantly reduces the fracture resistance in the four tested groups.

KEY WORDS: ceramics, fracture resistance, failure mode.

* Instructor of Fixed Prosthodontics, Faculty of Dentistry, Tanta University.

** Professor of Fixed Prosthodontics, Faculty of Dentistry, Tanta University.

*** Lecturer of Fixed Prosthodontics, Faculty of Dentistry, Tanta University. 


\section{INTRODUCTION}

Metal ceramic restorations have been widely used for fixed dental prosthesis. However, metal ceramic restorations have disadvantages, such as poor esthetics due to exposure of metal at the cervical margin, porcelain discoloration and debonding between the substructure and veneering material. They lead to staining of gingival tissue. These problems of metal ceramic restorations were resolved by the improvement of all-ceramic restorations.

All-ceramic usage in fixed prosthodontics has been increased thanks to its natural translucency and color, high biocompatibility, reasonable price compared to precious metals. As highstrength ceramic materials were developed due to the computer-aided design/computer-aided manufacturing (CAD/CAM) technologies, the usage of all ceramics in the posterior and anterior prosthesis has been expanded ${ }^{1}$.

Glass ceramics have high translucency, low thermal conductivity and high biocompatibility. On the other hand, they are brittle materials which is strong under compression but weak under tension, which may lead to failure in the oral environment ${ }^{2}$, ${ }^{3}$. They are fragile and brittle in case that they are manufactured too thin. They are only reliable to be used as restorations when fabricated in adequate thickness.

Lithium disilicate ceramic has higher flexural strength and fracture toughness than conventional silicate ceramics.

A second material is a lithium silicate which is reinforced with ten percent zirconia (ZLS-ceramic). Zirconia has the property of phase transformation where tetragonal phase transforms to the monoclinic phase leading to increase in volume which prevents crack propagation and resulting in higher fracture strength. Enhanced translucency and durability are combined in ZLS-ceramic ${ }^{4}$.
The yttria-stabilized tetragonal zirconia overcomes the problem of low tensile strength. As well as the material's good tissue tolerance and esthetics compared with PFM restorations, it has transformational toughening property which offers high flexural strength and fracture toughness. First studies found that a $0.5 \mathrm{~mm}$ thickness zirconia molar has sufficient fracture strength ${ }^{5}$.

However, due to the opaque appearance of zirconia, veneering material has been required with a high translucency like feldspathic porcelain to achieve the optimal esthetics ${ }^{6,7}$. To ensure the success of bilayered zirconia-based restorations, high bond strength is important between veneering ceramic material and the zirconia substructure.

Taskonak et $\mathrm{al}^{8}$ observed the causes of failure in bilayered zirconia-based restorations and found that the fractures were initiated in the ceramic veneering layer. Another study found that the bond between veneer and the core is the weakest point in the bilayered restoration ${ }^{9}$. To overcome this disadvantage, monolithic zirconia restorations, have been suggested ${ }^{10}$.

The ceramics characteristics significantly depend on prior thermal and mechanical fatigue loading. Ceramics deteriorate in the wet environment by slowly generating cracks, may be due to silicate bonds hydrolysis of ${ }^{11}$. These flaws are more aggravated by the stresses resulted from thermal variations within the oral cavity ${ }^{12}$.

So, this study compared fracture resistance and failure modes of lithium disilicate, zirconia reinforced lithium silicate, monolithic zirconia and bilayered zirconia in a crown design before and after thermocycling.

\section{MATERIALS AND METHODS}

In this in-vitro study, the total sample size was 64 crowns, (16 ceramic crowns in each group). The 16 samples of each ceramic group were further 
divided into 2 groups $(n=8)$, one group underwent no thermocycling and the other group underwent thermocycling. Group I: lithium disilicate (e.max CAD), group II: Zirconia reinforced lithium silicate (celtra duo), group III: monolithic zirconia (prettau zirconia), group IV: bilayered zirconia (zirconia substructure veneered with feldspathic porcelain).

Models of prepared nissin teeth \#14 (upper right first premolar) were used as abutments. The tooth model has standard preparation criteria for all ceramic crown. Abutments were mounted in an acrylic blocks. Each tooth was scanned by Identica digital scanner.

Designing the crowns was done with standard measurements: $1.5 \mathrm{~mm}$ at the buccal cusp and 2 $\mathrm{mm}$ at the palatal cusp by using ExoCad 2016 software. For group IV (bilayered zirconia), the design was adjusted to produce a $0.5 \mathrm{~mm}$-thick zirconia coping. Ceramic blocks were introduced to the VHF five axis milling machine. Wet milling was used for the lithium disilicate and zirconia reinforced lithium silicate blocks, while dry milling was used for zirconia blank. The restorations were Cleaned in an ultrasonic water bath and then dried. The final restorations were tried to examine their fit. Adjustments were done by using low speed water cooled grinding stones. The final restorations were tried to examine their fit.

Lithium disilicate crowns were crystallized in the Programat P310 Ivoclar Vivadent ceramic furnace. Zirconia restorations were sintered in the TABEO sintering oven. Then all monolithic restorations were glazed and stained to reach the desired shade. Then glaze fire was done according to manufacturer's instructions.

For group IV (bilayered zirconia), to standardize the veneering porcelain thickness and contour, a silicone index (ELITE HD, Zhermack) of a previously milled full anatomic monolithic lithium disilicate crown was made ${ }^{13}$. This index was used to guide the veneer build up to get standard thickness and external dimensions. The VITA VM 9 feldspathic porcelain was mixed and applied to the zirconia cores with layering technique then it was fired according to manufacturer's directions.

Zirconia fitting surface was airborne-particle abraded with $\mathrm{Al}_{2} \mathrm{O}_{3}(50 \mu)$. While for e.max CAD and Celtra Duo, the inner surface was etched with 5\% hydrofluoric acid gel (Vita Ceramic Etch) for 20 seconds and then rinsed with water and dried. Then silane coupling agent (Monobond N, Ivoclar Vivadent) was applied to the etched surface for one minute followed by air thinning. Self-adhesive resin cement (RelyX U200, 3M ESPE) was mixed and applied to the fitting surface of the crowns. Each restoration was seated on its corresponding abutment and fixed to a Universal Testing Machine for load application during cementation. A standard load of $5 \mathrm{Kg}(\cong 50 \mathrm{~N})$ was used. Tack cure was performed for 1-2 seconds per surface. Excess cement was removed using a scaler. Then each restoration was left for 6 minutes from start of mix for dark cure and complete setting. Excess cement was removed. The specimens were stored at room temperature in distilled water till they were tested.

Eight sample specimens from each group were thermocycled 500 times between the $55 \pm 1^{\circ} \mathrm{C}$ and 5 $\pm 1^{\circ} \mathrm{C}$ respectively, with a dwell time of 30 seconds in each bath and a lag time of 10 seconds. Fracture resistance was tested for the specimens before and after thermocycling using a computer controlled Universal Testing Machine. The load required to cause fracture was recorded in Newton.

One fractured specimen from each group was cleaned for 10 minutes in an ultrasonic alcohol bath. Then, the specimen was gold coated to be scanned with scanning electron microscopy (SEM). The microscope findings such as the crack origin and general crack propagation direction allowed for further analysis of the possible causes of failure. 


\section{RESULTS}

\section{Fracture resistance results}

Mean value for each group was calculated before and after thermal aging, and differences between groups were tested for statistical significance by use of one-way analysis of variance ANOVA test and Pair-wise Tukey's post-hoc test. Comparison of total fracture resistance results as function of thermal aging (effect of thermal aging on fracture resistance) was tested for statistical significance by use of two-way ANOVA test. P-value $<0.05$ was considered significant difference and $\mathrm{P}$-value $>0.05$ was considered non-significant.

Before thermocycling it was found that the highest fracture resistance mean value recorded with monolithic zirconia group followed by lithium disilicate group then bilayered zirconia group, while the lowest fracture resistance mean value recorded for zirconia reinforced lithium silicate group (1018.6 N) as shown in figure (1) and this was statistically significant. The four tested groups showed statistically significant lower fracture resistance mean values after thermal aging than before thermal aging.

\section{Failure mode results}

The fractography showed that surface cracks were the main origin of fractures in glass ceramic groups as shown in figure (2). While monolithic zirconia showed cementation internal surface cracks (radial cracks) as shown in figure (3).

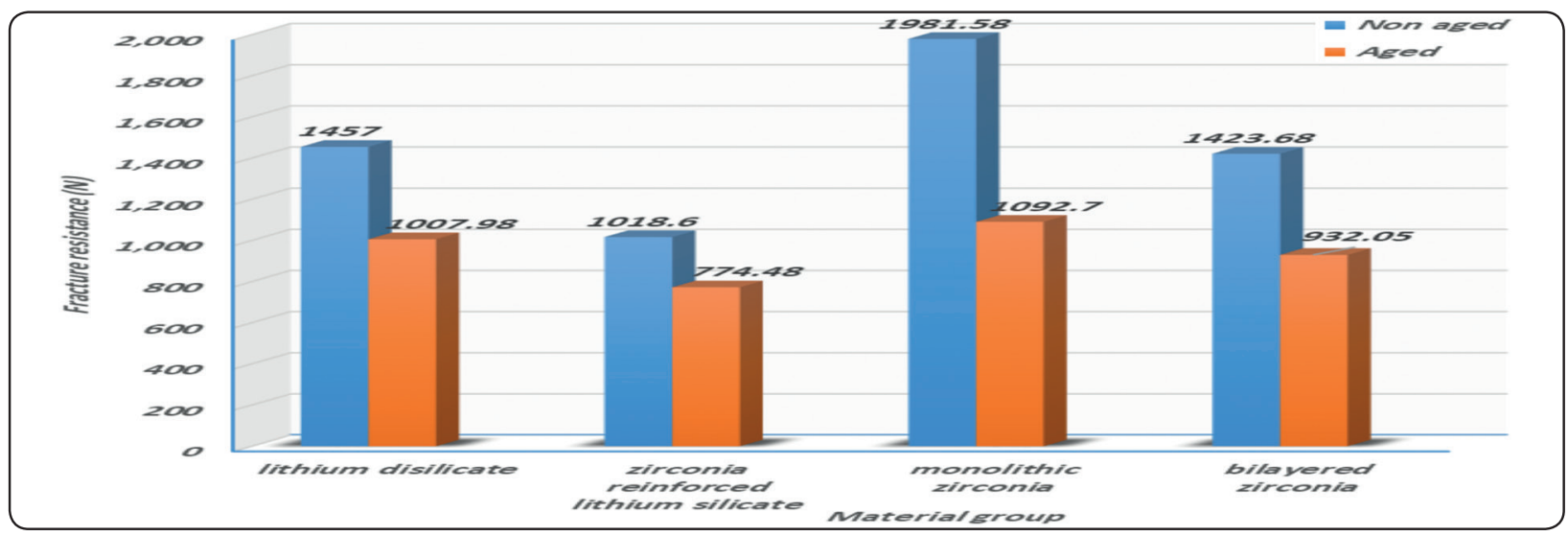

Fig. (1) Column chart comparing between fracture load mean values for before and after thermal aging for all groups.
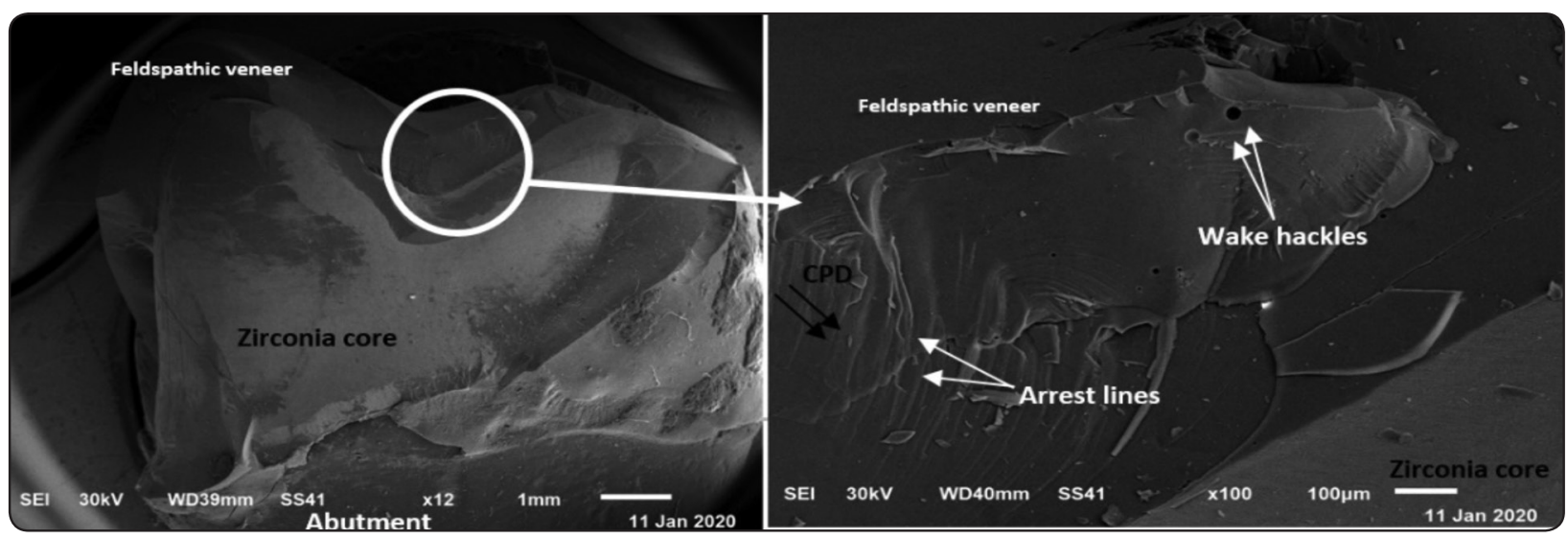

Fig. (2) fractured bilayered zirconia crown viewed at different magnifications under the SEM showing veneer chipping, arrest lines, wake hackles emanating from pores at the crack front, and crack propagation direction (CPD). 


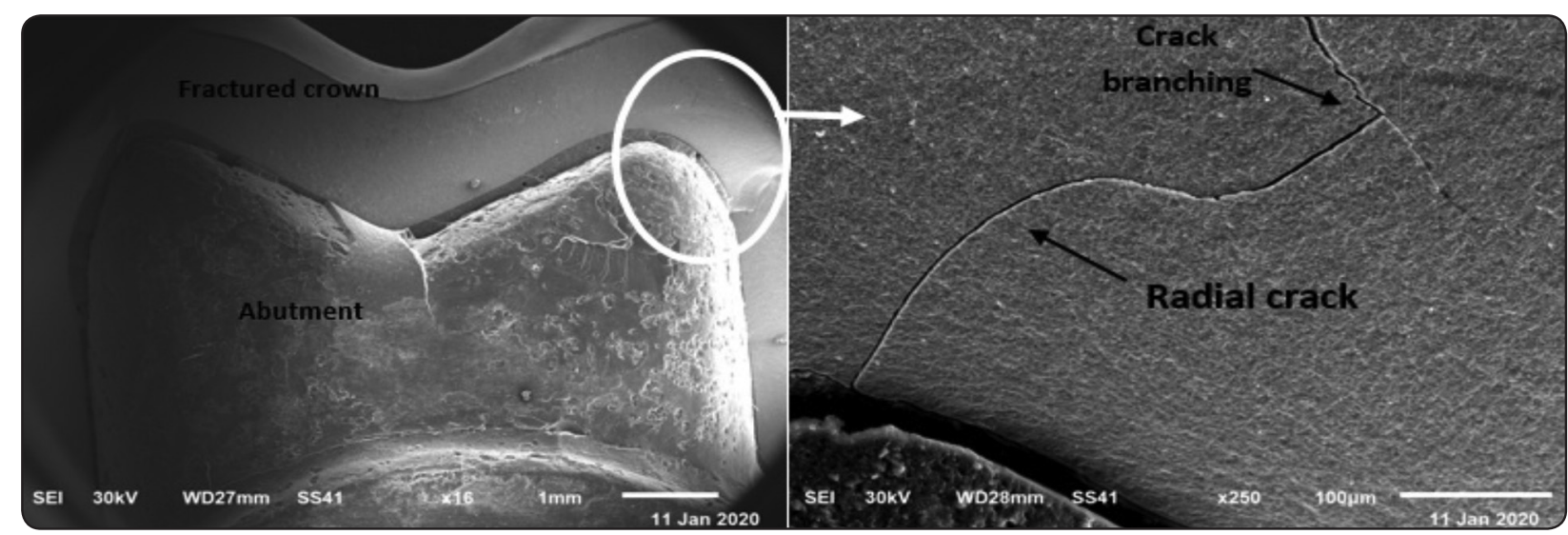

Fig. (3) monolithic zirconia crown part viewed at different magnifications under the SEM showing cementation internal surface radial cracks. Crack branching was also detected.

\section{DISCUSSION}

The aim of the present study was to compare the fracture resistance of lithium disilicate, zirconia reinforced lithium silicate, monolithic zirconia and bilayered zirconia.

The nissin dies were used to standardize the preparation and avoid variations in size in extracted natural teeth and also to avoid individual faults in teeth preparation. The elastic modulus of the dies should be close to that of the dentin, ranging from 10 to $15 \mathrm{GPa}$. The Nissin tooth is made of a homogeneous fiber reinforced plastic material. Its elastic modulus is about $10 \mathrm{GPa}^{14}$.

The highest fracture strength mean value before thermocycling was recorded with monolithic zirconia group $(1981.58 \mathrm{~N})$ as compared with crowns fabricated from other tested materials in the current study. This could be due to its polycrystalline structure where all atoms are packed into regular crystalline structures. So, it is more difficult for the crack to propagate than in irregular networks found in glasses. Another explanation is the transformation toughening mechanism where stress at the front of the crack induces the transformation of the tetragonal phase to the monoclinic phase, resulting in volume expansion that creates a compressive stress on the developing crack and prevents its propagation.

The second highest fracture resistance mean value before thermocycling was recorded with lithium disilicate group $(1457 \mathrm{~N})$ which was followed by bilayered zirconia group (1423.68 N). This may be because of low strength of the ceramic veneer or the weak bond between the core and the veneer. These findings are in agreement with previous studies, which stated that the mechanical strength of bilayered zirconia is limited to the weak feldspathic veneer ${ }^{15}$. These findings were confirmed in this study by electron microscopic examination, where the concave orientation of the arrest lines of the chipped veneering layer indicated that the damage started on the occlusal surface of the crown.

Both the lithium disilicate and zirconia-reinforced lithium silicate crowns underwent unfavorable fracture with complete restoration fracture, while the bilayered zirconia crowns underwent a more favorable failure, which is a repairable chipping of the veneering feldspathic.

The fracture resistance mean value recorded by lithium disilicate group $(1457 \mathrm{~N})$ was also higher than that of zirconia reinforced lithium silicate group $(1018.6 \mathrm{~N})$. These results are not in agreement with results of Elsaka et al. ${ }^{13,16}$ as they found that the fracture toughness and flexural strength of zirconia reinforced lithium silicate were higher than those of lithium disilicate. Though the manufacturer advocates that the addition of zirconia to ZLS composition increases strength, a previous study could not verify this ${ }^{17}$. The results of the current 
study agree with other studies ${ }^{17-19}$. Wendler ${ }^{18}$ examined both brands of zirconia reinforced lithium silicate, Vita suprinity and Celtra duo, and found a lower strength values than other tested groups. He suggested that the lower fracture strength of zirconia reinforced lithium silicate is due to a severe incompatibility in coefficient of thermal expansion between phases (three crystal phases + one glass phase containing dissolved $\mathrm{ZrO}_{2}$ ), leading to residual stresses during cooling which lead to micro-cracking.

Thermocycling can simulate the oral cavity environment. So, it is used as an artificial aging process. There is a large variation in the number of cycles and in the temperature extremes between studies. So, the International Organization for Standardization (ISO) made standard protocol for thermocycling tests to enable investigators to interpret and compare results. According to this protocol, 500 cycles in water between $5^{\circ}$ and $55^{\circ} \mathrm{C}$ is an appropriate artificial aging test ${ }^{20}$.

The four tested groups showed statistically significant lower fracture resistance mean values after thermal aging than before thermal aging. This is in agreement with a previous study ${ }^{21}$. This may be due to abrupt changes of temperature to bonded different materials, die material and ceramic crown, which have different thermal expansion coefficients resulting in thermal stresses at the interface. The thermal stresses may result in tension that facilitates crack initiation within the ceramic crowns, leading to catastrophic fracture ${ }^{22}$. Another explanation is that ceramics deteriorate in the wet environment by slowly advancing cracks, which result from hydrolysis of silicate bonds. These cracks are more aggravated by the stresses induced by thermal changes within the oral cavity ${ }^{11-13}$.

The fractography showed that surface cracks were the main origin of fractures in glass ceramic groups. The cracks propagated from the occlusal surface downward to the inner surface. This may be due to the microstructure of the weaker glass ceramics. While monolithic zirconia showed cementation internal surface cracks (radial cracks). This may be due to higher elastic modulus of zirconia compared to other groups. According to Lawn ${ }^{23}$, The critical load required to initiate radial crack depends on the elastic modulus difference between the supporting structure material and the crown material. The elastic modulus difference between the crown and the supporting die is higher in case of monolithic zirconia crown which showed internal surface cracks.

Another finding was crack deflection that was detected in lithium disilicate crown. This could be due to incorporated crystal particles within glassy ceramic matrix that can deflect cracks dissipating fracture energy and improve fracture toughness ${ }^{24}$. On the other hand, crack branching was observed in monolithic zirconia crown. According to El Attaoui et al. ${ }^{25}$, crack branching could be a result of phase transformation, where the main crack is arrested and a minor crack is initiated in the tensile region.

The limitations of the current study were that it was in vitro study and fracture resistance test was accomplished on nissin dies, not on natural teeth, which may have led to different results due to absence of true bonding and also different elastic modulus from dentine. Another limitation was that the specimens were subjected to thermal aging only, which cannot perfectly simulate the oral environment where restorations are subjected to both thermal and mechanical aging. So, long-term clinical studies are needed to provide reliable data.

\section{CONCLUSIONS}

Within limitations of this study, it could be concluded that Crowns of all groups revealed fracture strength above the clinically expected occlusal forces. Monolithic zirconia has the highest fracture resistance among the tested groups, followed by lithium disilicate group then bilayered zirconia group, while the lowest fracture resistance was recorded for zirconia reinforced lithium silicate group. Thermal aging significantly reduces the fracture resistance in the four tested groups. Surface defects are the main origin of fractures in glass ceramic groups. While in monolithic zirconia, internal surface cracks (radial cracks) are the main origin of fractures. 


\section{REFERENCES}

1. Schultheis S, Strub JR, Gerds TA, Guess PC. Monolithic and bi-layer CAD/CAM lithium-disilicate versus metalceramic fixed dental prostheses: comparison of fracture loads and failure modes after fatigue. Clin Oral Investig 2013;17(5):1407-13.

2. Hamza TA, Ezzat HA, El-Hossary MM, et al. Accuracy of ceramic restorations made with two CAD/CAM systems. J Prosthet Dent 2013;109(2):83-7.

3. Kang SH, Chang J, Son HH. Flexural strength and microstructure of two lithium disilicate glass ceramics for CAD/ CAM restoration in the dental clinic. Restor Dent Endod 2013;38(3):134-40.

4. Manicone PF, Rossi Iommetti P, Raffaelli L. An overview of zirconia ceramics: basic properties and clinical applications. J Dent 2007;35(11):819-26.

5. Nakamura K, Harada A, Inagaki R, et al. Fracture resistance of monolithic zirconia molar crowns with reduced thickness. Acta Odontol Scand 2015;73(8):602-8.

6. Guazzato M, Albakry M, Ringer SP, Swain MV. Strength, fracture toughness and microstructure of a selection of allceramic materials. Part II. Zirconia-based dental ceramics. Dent Mater 2004;20(5):449-56.

7. Jurisic S, Jurisic G, Zlataric DK. In Vitro Evaluation and Comparison of the Translucency of Two Different All-Ceramic Systems. Acta Stomatol Croat 2015;49(3):195-203.

8. Taskonak B, Borges GA, Mecholsky JJ, Jr., et al. The effects of viscoelastic parameters on residual stress development in a zirconia/glass bilayer dental ceramic. Dent Mater 2008;24(9):1149-55.

9. Kanat B, Comlekoglu EM, Dundar-Comlekoglu M, et al. Effect of various veneering techniques on mechanical strength of computer-controlled zirconia framework designs. J Prosthodont 2014;23(6):445-55.

10. Rauch A, Reich S, Schierz O. Chair-side generated posterior monolithic lithium disilicate crowns: clinical survival after 6 years. Clinical oral investigations 2017;21(6):2083-89.

11. Magne P, Kwon K-R, Belser UC, Hodges JS, Douglas WH. Crack propensity of porcelain laminate veneers: a simulated operatory evaluation. The Journal of prosthetic dentistry 1999;81(3):327-34.

12. Anusavice K, Lee R. Effect of firing temperature and water exposure on crack propagation in unglazed porcelain. Journal of Dental Research 1989;68(6):1075-81.
13. Hamza TA, Sherif RM. Fracture Resistance of Monolithic Glass-Ceramics Versus Bilayered Zirconia-Based Restorations. Journal of Prosthodontics 2019; 28(1): e259-e64.

14. He L-H, Foster Page L, Purton D. An evaluation of dental operative simulation materials. Dental materials journal 2012;31:645-9.

15. Manicone PF, Iommetti PR, Raffaelli L. An overview of zirconia ceramics: basic properties and clinical applications. Journal of dentistry 2007;35(11):819-26.

16. Elsaka SE, Elnaghy AM. Mechanical properties of zirconia reinforced lithium silicate glass-ceramic. Dent Mater 2016;32(7):908-14.

17. Ramos Nde C, Campos TM, Paz IS, et al. Microstructure characterization and SCG of newly engineered dental ceramics. Dent Mater 2016;32(7):870-8.

18. Wendler M, Belli R, Petschelt A, et al. Chairside CAD/ CAM materials. Part 2: Flexural strength testing. Dent Mater 2017;33(1):99-109.

19. Nishioka G, Prochnow C, Firmino A, et al. Fatigue strength of several dental ceramics indicated for CAD-CAM monolithic restorations. Braz Oral Res 2018;32:e53.

20. Morresi AL, D'Amario M, Capogreco M, et al. Thermal cycling for restorative materials: does a standardized protocol exist in laboratory testing? A literature review. J Mech Behav Biomed Mater 2014;29:295-308.

21. Yang R, Arola D, Han Z, Zhang X. A comparison of the fracture resistance of three machinable ceramics after thermal and mechanical fatigue. The Journal of prosthetic dentistry 2014;112(4):878-85.

22. Kern M, Wegner SM. Bonding to zirconia ceramic: adhesion methods and their durability. Dent Mater 1998;14(1):64-71.

23. Lawn BR. Ceramic-based layer structures for biomechanical applications. Current opinion in solid state and materials science 2002;6(3):229-35.

24. Ma L. Fundamental formulation for transformation toughening. International Journal of Solids and Structures 2010;47(22-23):3214-20.

25. El Attaoui H, Saâdaoui M, Chevalier J, Fantozzi G. Static and cyclic crack propagation in Ce-TZP ceramics with different amounts of transformation toughening. Journal of the European Ceramic Society 2007;27(2-3):483-86. 